\title{
Complete Response to Erlotinib and Bevacizumab in a Patient With Biphenotypic (Hepatobiliary) Primary Liver Carcinoma
}

\author{
Amy Zhou, MD; ; Manik Amin, MDa; Kathryn J. Fowler, MD ; Elizabeth M. Brunt, MDc; Jesse Keller, MD; \\ and Benjamin Tan, MDa
}

\section{Abstract}

Biphenotypic (hepatobiliary) primary liver carcinomas [B(H-B)PLCs] are rare tumors with features of both hepatocellular carcinoma (HCC) and intrahepatic cholangiocarcinoma (ICC). These tumors are associated with a poor overall prognosis and treatment is not well defined. Research over the past 20 years has identified aberrations in several molecular pathways, including epidermal growth factor receptor (EGFR) and vascular endothelial growth factor (VEGF) in hepatocellular and biliary tract cancers. These discoveries led to the evaluation of targeted therapies, such as tyrosine kinase inhibitors, for the treatment of HCC and ICC. We report a case of a patient with metastatic $\mathrm{B}(\mathrm{H}-\mathrm{B}) \mathrm{PLC}$ found to have a single nucleotide variant in the EGFR gene locus R521K who achieved a complete response on imaging after treatment with the combination of an EGFR inhibitor and a VEGF inhibitor. This case prompts consideration of further genomic analysis of these rare tumors and the potential use of targeted therapies in the treatment of patients with B(H-B)PLCs. (J Natl Compr Canc Netw 2015;13:1468-1473)

Biphenotypic (hepatobiliary) primary liver carcinomas [B(H-B)PLCs] are rare tumors representing a heterogenous group of primary liver malignancies with evidence of both biliary and hepatocellular differentiation. These tumors have been referred to by several other names, including hepatocholangiocellular carcinoma, mixed hepatocellular cholangiocarcinoma, and combined hepatocellular cholangiocarcinoma. These tumors account for approximately $1 \%$ to $14 \%$ of all primary liver cancers. ${ }^{1}$ $\mathrm{B}(\mathrm{H}-\mathrm{B}) \mathrm{PLCs}$ have been increasingly recognized as distinct from hepatocellular carcinomas (HCCs) and intrahepatic cholangiocarcinomas (ICCs) since the first report in 1903 by Wells ${ }^{2}$ and further characterization by Allen and $\mathrm{Lisa}^{3}$ in 1949. In general, patients with B(HB)PLCs tend to have a worse prognosis than those with HCCs, ${ }^{4,5}$ with overall survival (OS) outcomes similar to

From the aDepartment of Medicine, 'Department of Radiology, and 'Department of Pathology, Washington University School of Medicine, St. Louis, Missouri.

Submitted June 26, 2015; accepted for publication September 25, 2015.

Drs. Zhou, Amin, Fowler, and Keller have no financial interests, arrangements, affiliations, or commercial interests with the manufacturers of any products discussed in this article or their competitors. Dr. Brunt has disclosed that she is the co-moderator of the International Pathology Consensus Consortium. Dr. Tan receives grant/research support from Genentech.

Correspondence: Amy Zhou, MD, Washington University School of Medicine, 660 South Euclid Avenue, Campus Box 8056, St. Louis,

MO 63110. E-mail: azhou@DOM.wustl.edu those of ICCs; however, this is controversial, because studies have shown variability in outcomes, likely due to small sample sizes. ${ }^{6,7}$ No clear guidelines exist regarding the management of $\mathrm{B}(\mathrm{H}-\mathrm{B}) \mathrm{PLCs}$. Hepatic resection remains the preferred treatment; however, for patients with unresectable lesions, locoregional or systemic therapy is considered. HCCs and ICCs respond poorly to chemotherapy, and novel targeted agents are currently being explored in the treatment of these tumors.

This article reports a case of a patient with metastatic $\mathrm{B}(\mathrm{H}-\mathrm{B}) \mathrm{PLC}$ who experienced a complete response on imaging after treatment with the combination of an epidermal growth factor receptor (EGFR) inhibitor and a vascular endothelial growth factor (VEGF) inhibitor.

\section{Case Presentation}

During a workup for chest pain in a 77-year-old Caucasian woman, a 3-cm liver lesion was found incidentally. CA19-9, carcinoembryonic antigen (CEA), and $\alpha$-fetoprotein (AFP) were all within normal limits and hepatitis serologies were negative. She subsequently underwent a biopsy, which showed large cells with abundant eosinophilic cytoplasm and nuclear hyperchromasia forming glands and trabecular structures in an extensively hyalinized stroma. Immunohistochemical (IHC) studies showed strong immunoreactivity to 

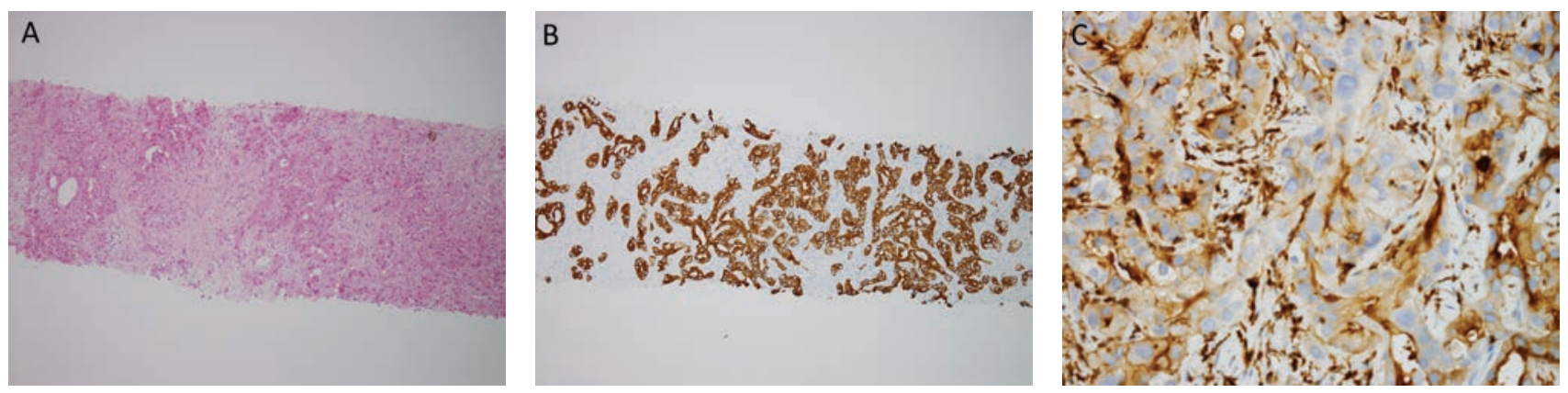

Figure 1 Histopathologic examination of biopsy specimen. (A) Hematoxylin and eosin staining shows poorly formed glands (origina magnification $\times 20$ ). (B) Kinase 19 immunostaining shows diffuse reactivity (original magnification $\times 20$ ). (C) Polyclonal carcinoembryonic antigen staining shows canalicular reactivity (original magnification $\mathrm{x} 40$ ).

keratin 7 (K7) and keratin 19 (K19), and polyclonal CEA showed variable reactivity, including abundant tumor cells with canalicular formation consistent with a diagnosis of biphenotypic hepatocellular carcinoma/cholangiocarcinoma (Figure 1). She underwent a segment 4 and 5 hepatic resection with negative surgical margins; however, there was evidence of tumor extension through the liver capsule and a pericystic duct lymph node was positive for tumor involvement, consistent with stage IV disease.

She was seen by Medical Oncology and underwent 8 cycles of adjuvant chemotherapy with singleagent gemcitabine. Six months after completion of adjuvant therapy, she was found to have peritoneal lesions concerning for recurrent metastatic disease on abdominal MRI (Figure 2A). Next-generation sequencing of her tumor from the initial biopsy demonstrated a single nucleotide variant in the EGFR gene locus $\mathrm{R} 521 \mathrm{~K}$. This polymorphism is of unclear clinical significance in hepatobiliary tract malignancies, but prompted consideration for treatment with an EGFR inhibitor. She was subsequently started on erlotinib, an anti-EGFR small tyrosine kinase inhibitor, at $150 \mathrm{mg}$ orally daily and bevacizumab at $15 \mathrm{mg} / \mathrm{kg}$ intravenously every 3 weeks. She tolerated treatment well, with minimal toxicity, and a repeat MRI after 7 cycles of therapy showed complete resolution of the previously seen peritoneal lesions (Figure 2B). She continued on therapy for an additional 2 months and a repeat MRI again showed no radiologic evidence of disease.

She was subsequently given a treatment break, with no evidence of disease recurrence on repeat MRI following 6 months off-therapy. A month after that scan, however, she developed increasing ascites and abdominal pain. Workup revealed recurrent diffuse omental metastases. She was then restarted on erlotinib and bevacizumab, with prompt resolution of her ascites and abdominal discomfort. Bevacizumab was subsequently held a month after retreatment because of hypertension and chest pain, but she was continued on erlotinib at $150 \mathrm{mg}$ daily alone. A repeat MRI after 3 months of therapy showed stable omental disease. She has continued on single-agent erlotinib for almost a year, with imaging continuing to show stable disease and no evidence of new me-
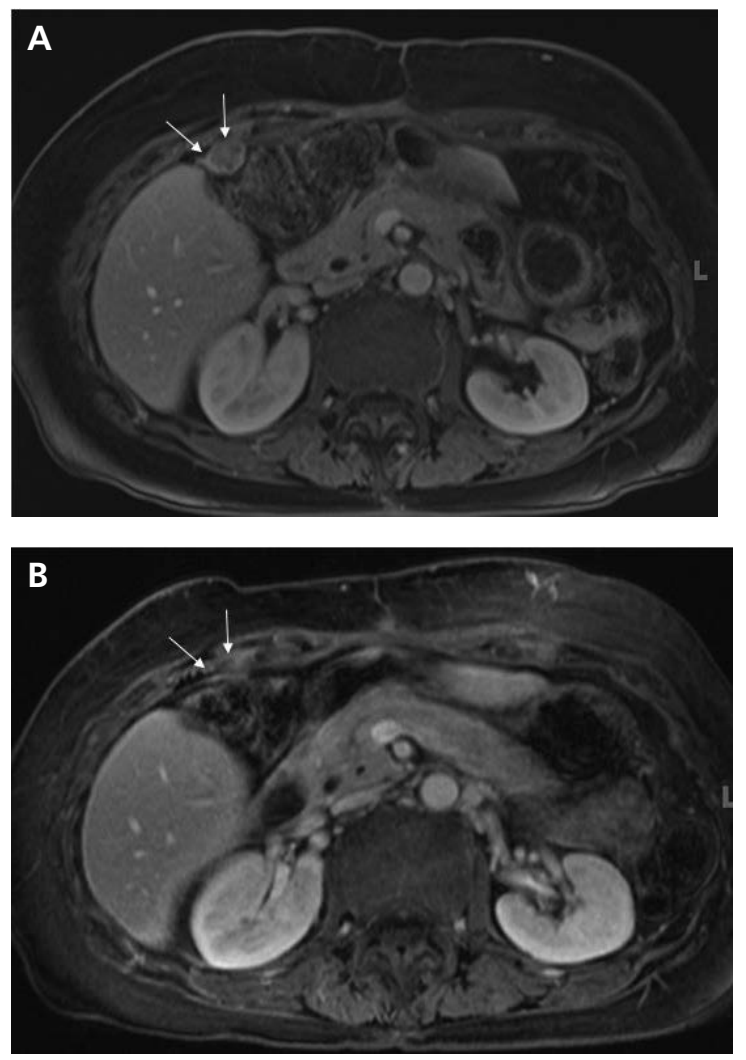

Figure 2 MRI imaging pretreatment and posttreatment. (A) Initial postoperative MRI images show an enhancing soft tissue peritoneal nodule (arrows) compatible with metastases. (B) Follow-up imaging after 3 months of treatment shows complete resolution of the nodule. 
tastases. At the time of this report, 42 months have elapsed from the time of initial diagnosis and 27 months since the time of metastatic disease.

\section{Discussion}

The WHO defines B(H-B)PLCs as tumors containing unequivocal elements of both HCC and ICC, which are intimately admixed, ${ }^{8}$ with further subtypes, including those with stem cell features. These tumors should be distinguished from cases of separate HCC and ICC that arise in the same liver or collision-type tumors in which HCC and ICC are present at adjacent sites. For a diagnosis of B(H-B)PLC to be made, the tumor must have IHC features of biliary (K7, K19) and hepatocellular (eg, polyclonal CEA [pCEA] and CD10 with canalicular staining, cytoplasmic hepatocyte paraffin 1 [HepPar1], thyroid transcription factor-1 [TTF-1]) differentiation, although it is important to note that not all of the IHC markers are consistently positive. ${ }^{9-12}$

The cell or cells of origin in B(H-B)PLC tumors are unknown. It is postulated that the biphenotypic features may be derived from liver progenitor cells or dedifferentiation of mature hepatocytes or HCC. Several studies have shown that HCC and ICC may originate from stem cells $\mathrm{s}^{13,14}$ and that B(H-B)PLC tumor cells display staining for liver progenitor (K19, epithelial call adhesion molecule [epCAM]) and/or stem cell (CD133, CD44) markers, ${ }^{15,16}$ supporting the former theory. Comparison of gene expression profiling in HCC, ICC, and B(H-B)PLC tumors found that HCC and ICC groups could be clearly distinguished from one another. ${ }^{17}$ Interestingly, more than $70 \%$ of $\mathrm{B}(\mathrm{H}-\mathrm{B}) \mathrm{PLC}$ tumors clustered with the ICC group, suggesting a closer association of $\mathrm{B}(\mathrm{H}-\mathrm{B}) \mathrm{PLC}$ to ICC at the gene expression level.

The clinical presentation of $\mathrm{B}(\mathrm{H}-\mathrm{B}) \mathrm{PLC}$ may be similar to HCCs and ICCs, with incidental finding of a liver mass on imaging or symptoms of abdominal pain, jaundice, ascites, fatigue, weight loss, cholangitis, or pruritus with more advanced disease. Serum tumor markers, such as CA 19-9 or AFP, are not specific for $\mathrm{B}(\mathrm{H}-\mathrm{B}) \mathrm{PLC}$ and would not differentiate them from HCCs or ICCs; however, B(H-B)PLCs may be suspected if both are simultaneously elevated or there is discordance between tumor marker elevation and imaging findings. The etiologic, epidemiologic, and clinical features of $\mathrm{B}(\mathrm{H}-\mathrm{B}) \mathrm{PLCs}$ that have been described are variable and may differ by geographic region. The association with chronic liver disease and cirrhosis, for example, may differ between Eastern and Western populations. Case reports from Asia demonstrate that B(H-B)PLCs are more frequently observed in older male patients with chronic hepatitis (more frequently hepatitis B) or cirrhosis, ${ }^{7,18,19}$ whereas in contrast, the few US case reports demonstrated no significant difference in gender distribution, and patients were frequently without chronic liver disease, including in our own institutional experience. . $^{1,20}$

A recent review of the world's literature summarizes clinical, histologic, and molecular findings up to the current time. ${ }^{21}$ One important concept is that, although $\mathrm{B}(\mathrm{H}-\mathrm{B}) \mathrm{PLC}$ may be classified under a single rubric, this is not a single entity and these tumors have several different phenotypes. Thus, identification of histopathologic features often requires application of various IHC stains on otherwise "typical" HCCs or ICCs. This feature may be one of the reasons for differing outcomes among series.

Dynamic contrast-enhanced MRI and CT are the preferred imaging modalities in the evaluation of $\mathrm{B}(\mathrm{H}-\mathrm{B}) \mathrm{PLCs}$. MRI may have greater sensitivity than $\mathrm{CT}$ and be more preferable given the absence of ionizing radiation and superior contrast resolution. ${ }^{22,23}$ The imaging appearances of B(H-B)PLCs may display overlap between HCC and ICC. Characteristic findings of HCC on CT or MRI include arterial enhancement with washout on portal venous or equilibrium phase imaging, and an enhancing pseudocapsule on delayed images, whereas characteristic findings of ICC include peripheral arterial rim enhancement with progressive centripetal enhancement of fibrous stroma, capsular retraction, and associated biliary ductal dilatation. ${ }^{22}$ A case series report from our institution demonstrated that many of the $\mathrm{B}(\mathrm{H}-\mathrm{B}) \mathrm{PLC}$ tumors more closely resembled ICC on imaging, ${ }^{20}$ and similar findings were observed in a recently published retrospective case series from the Mayo Clinic. ${ }^{24}$

Treatment of B(H-B)PLCs is not well defined given the rarity of these tumors, and current treatment modalities have been extrapolated from the treatment of HCCs or ICCs, which may include hepatic resection, liver transplantation, transarterial chemoembolization, local radiofrequency ablation, or chemotherapy, depending on the stage of the 
Targeted Therapy in Biphenotypic Tumors

disease. The prognosis for $\mathrm{B}(\mathrm{H}-\mathrm{B}) \mathrm{PLCs}$ is generally worse compared with that of HCCs and ICCs, with most studies demonstrating decreased OS. 1,5,7,25 Our own institutional experience has found that patients with $\mathrm{B}(\mathrm{H}-\mathrm{B}) \mathrm{PLC}$ have an OS of $39.2 \%$ at 1 year, with the presence of tumor thrombus and satellite lesions being the strongest predictors of poor survival. ${ }^{26}$ A case series on liver transplantation for these rare tumors demonstrated a high 5 -year recurrence rate of $78 \%$ compared with only $17 \%$ in patients with HCC. ${ }^{27}$ Hepatic resection may offer patients the longest OS; however, the risk of recurrence is high even after resection, with a median time to recurrence of 6 to 9 months. ${ }^{7}$ Our institutional study of 65 patients with $\mathrm{B}(\mathrm{H}-\mathrm{B}) \mathrm{PLC}$ found that male sex and the presence of satellite lesions were strongly predictive of disease recurrence after resection. ${ }^{26} \mathrm{HCC}$ generally does not respond to chemotherapy, and although gemcitabine-based regimens have demonstrated improved survival in patients with advanced ICC, the median OS is still less than 1 year. ${ }^{28}$ Data for the use of chemotherapy in patients with advanced $\mathrm{B}(\mathrm{H}-\mathrm{B})$ PLC are limited to a few case reports, with a recent case demonstrating disease control for approximately 12 months with gemcitabine and cisplatin. ${ }^{29}$

Over the past 20 years, research has identified aberrations in several molecular pathways in hepatocellular and biliary tract cancers, which has led to the evaluation of targeted therapies such as tyrosine kinase inhibitors for the treatment of HCC and cholangiocarcinoma. Sorafenib, an oral multikinase inhibitor of VEGF receptor, platelet-derived growth factor receptor, and Raf, has demonstrated improvement in median OS (10.7 months) compared with placebo (7.9 months) in patients with advanced HCC, leading to its subsequent FDA approval..$^{30}$ EGFR is expressed in most biliary tract cancers, ${ }^{31}$ and overexpression, sustained activation, and mutation have been described in human cholangiocarcinoma cells. ${ }^{32}$ This led to the evaluation of EGFR inhibitors in the treatment of patients with advanced biliary cancers. In a phase II study of 42 patients with advanced biliary tract cancers, erlotinib at $150 \mathrm{mg}$ daily demonstrated a median OS of 7.5 months and median time to disease progression of 2.6 months, although no conclusions could be made regarding the correlation of EGFR expression to disease response given the small sample size. ${ }^{33}$ Subsequently, a phase III randomized controlled trial with gemcitabine and oxaliplatin with or without erlotinib was conducted, which demonstrated no difference in median progression-free survival (PFS) or OS between the cohorts. ${ }^{34}$ A similar study was conducted using another EGFR inhibitor, cetuximab, which also failed to demonstrate an improvement in PFS or OS..$^{35}$ The significance of the EGFR R $521 \mathrm{~K}$ polymorphism in $\mathrm{B}(\mathrm{H}-\mathrm{B}) \mathrm{PLC}$ or any other cancer is still unclear. This particular EGFR polymorphism, located in the CT2 domain of the EGFR gene, has been associated with improved outcomes with anti-EGFR therapy use in colorectal and head and neck cancers. ${ }^{36-38}$ The EGFR R $521 \mathrm{~K}$ polymorphism seen in our patient was thought to be most consistent with a constitutional variant.

VEGF is a key factor of angiogenesis that facilitates tumor growth and metastasis. It is expressed in approximately $30 \%$ to $50 \%$ of biliary tract cancers and may correlate with a worse prognosis. ${ }^{39}$ Bevacizumab, a VEGF inhibitor, has been evaluated in advanced biliary tract cancers in combination with erlotinib and chemotherapy. In a small phase II study of 53 patients with unresectable biliary tract cancers, the combination of erlotinib at $150 \mathrm{mg}$ daily with bevacizumab at $5 \mathrm{mg} / \mathrm{kg}$ every 2 weeks was well tolerated with $12 \%$ of patients demonstrating a partial response at a median duration of 8.4 months and $51 \%$ of patients demonstrated stable disease..$^{40}$ The combination of bevacizumab with gemcitabine and oxaliplatin demonstrated an overall response rate of $41 \%$ with a median PFS of 7.6 months and median OS of 14.2 months for patients with ICC. ${ }^{41}$

The use of next-generation sequencing has helped to identify additional targetable pathways in patients with biliary tract tumors. A recent $a b$ stract presented by Ross et $\mathrm{al}^{42}$ at the Gastrointestinal Cancers Symposium reported the results of next-generation sequencing on 554 biliary tract cancer specimens, including patients with ICC, extrahepatic cholangiocarcinoma (ECC), and gallbladder cancer. Interestingly, two-thirds of patients were found to harbor a clinically relevant genomic alteration. ICCs demonstrated increased FGFR1-3 fusions and amplifications and IDH1/2 substitutions compared with ECC and gallbladder cancers, whereas all 3 malignancies shared genomic alterations in cell cycle (CDKN2A/B loss) and chromatin remodeling (ARID1A alterations). The abstract described 4 patients who were found individually to have al- 
terations in ERBB2, FGFR3, BRAF, and EGFR and who achieved partial clinical response to treatment with their respective inhibitors, but none showed a complete remission on imaging. Genomic analysis via targeted next-generation sequencing of 18 $\mathrm{B}(\mathrm{H}-\mathrm{B}) \mathrm{PLC}$ patient samples from our institutional biliary registry revealed high rates of mutations in p53, FGFR4, FLT3, and EGFR, and a statistically significant increase in $p 53$ mutations was observed in $\mathrm{B}(\mathrm{H}-\mathrm{B}) \mathrm{PLC}$ tumors compared with HCC and CC. ${ }^{43}$ The incidence of $p 53$ mutations in $\mathrm{B}(\mathrm{H}-\mathrm{B}) \mathrm{PLC}$ tumors has previously been reported at $10 \%$ to $29 \% .{ }^{44}$ Additionally, loss of heterozygosity in $4 \mathrm{q}, 8 \mathrm{p}, 13 \mathrm{q}$, $16 \mathrm{q}$, and $17 \mathrm{p}$ are frequently seen in B(H-B)PLCs, along with $3 \mathrm{p}$ and $14 \mathrm{q}$, and mutations in KRAS have also been rarely described. ${ }^{44}$

To our knowledge, this case represents the only report of a patient with $\mathrm{B}(\mathrm{H}-\mathrm{B}) \mathrm{PLC}$ who experienced a complete response to targeted therapy with EGFR and VEGF inhibitors. Whether the presence of the EGFR R $521 \mathrm{~K}$ polymorphism contributed to the patient's dramatic response to targeted therapy is unclear, because it is likely to represent a germline single nucleotide polymorphism rather than a driver mutation. Therefore, the reason for the patient's dramatic response to targeted therapy and prolonged survival has yet to be elucidated.

\section{Conclusions}

$\mathrm{B}(\mathrm{H}-\mathrm{B}) \mathrm{PLC}$ are heterogenous, rare tumors that are distinct from HCCs and ICCs and are associated with a poor overall prognosis. Treatment of $\mathrm{B}(\mathrm{H}-\mathrm{B}) \mathrm{PLC}$ is not well defined, but the use of targeted therapies may prove to be effective, as illustrated in this case. Further research and improved understanding of the disease pathogenesis may ultimately improve the treatment and prognosis of these cancers.

\section{References}

1. Jarnagin WR, Weber S, Tickoo SK, et al. Combined hepatocellular and cholangiocarcinoma: demographic, clinical, and prognostic factors. Cancer 2002;94:2040-2046.

2. Wells H. Primary carcinoma of the liver. Am J Sci 1903;126:403-417.

3. Allen RA, Lisa JR. Combined liver cell and bile duct carcinoma. Am J Pathol 1949;25:647-655.

4. Maeda $T$, Adachi E, Kajiyama K, et al. Combined hepatocellular and cholangiocarcinoma: proposed criteria according to cytokeratin expression and analysis of clinicopathologic features. Hum Pathol 1995;26:956-964.

5. Koh KC, Lee H, Choi MS, et al. Clinicopathologic features and prognosis of combined hepatocellular cholangiocarcinoma. Am J Surg 2005;189:120-125.
6. Lee JH, Chung GE, Yu SJ, et al. Long-term prognosis of combined hepatocellular and cholangiocarcinoma after curative resection comparison with hepatocellular carcinoma and cholangiocarcinoma. J Clin Gastroenterol 2011;45:69-75

7. Yin $\mathrm{X}$, Zhang BH, Qiu SJ, et al. Combined hepatocellular carcinoma and cholangiocarcinoma: clinical features, treatment modalities, and prognosis. Ann Surg Oncol 2012;19:2869-2876.

8. Theise ND, Nakashima O, Park YN, Nakanuma Y. Combined hepatocellular-cholangiocarcinoma. In: Bosman FT, Carneiro F, Hruban RH, Theise ND, eds. WHO classification of tumours of the digestive system. 4th ed. Lyon, France: International Agency of Research on Cancer; 2010:225-227.

9. Itoyama M, Hata M, Yamanegi K, et al. Expression of both hepatocellular carcinoma and cholangiocarcinoma phenotypes in hepatocellular carcinoma and cholangiocarcinoma components in combined hepatocellular and cholangiocarcinoma. Med Mol Morphol 2012;45:7-13.

10. Al-Muhannadi N, Ansari N, Brahmi U, Satir AA. Differential diagnosis of malignant epithelial tumours in the liver: an immunohistochemical study on liver biopsy material. Ann Hepatol 2011;10:508-515.

11. Lei JY, Huang J. Cytoplasmic staining of TTF-1 in the differential diagnosis of hepatocellular carcinoma. Expert Opin Med Diagn 2008;2:151-159.

12. Zhang F, Chen XP, Zhang W, et al. Combined hepatocellular cholangiocarcinoma originating from hepatic progenitor cells: immunohistochemical and double-fluorescence immunostaining evidence. Histopathology 2008;52:224-232.

13. Reya T, Morrison SJ, Clarke MF, Weissman IL. Stem cells, cancer, and cancer stem cells. Nature 2001;414:105-111.

14. Alison MR. Liver stem cells: implications for hepatocarcinogenesis. Stem Cell Rev 2005;1:253-260.

15. Yu XH, Xu LB, Zeng $H$, et al. Clinicopathological analysis of 14 patients with combined hepatocellular carcinoma and cholangiocarcinoma. Hepatobiliary Pancreat Dis Int 2011;10:620-625.

16. Coulouarn C, Cavard C, Rubbia-Brandt L, et al. Combined hepatocellularcholangiocarcinomas exhibit progenitor features and activation of Wnt and TGFbeta signaling pathways. Carcinogenesis 2012;33:1791-1796.

17. Woo HG, Lee JH, Yoon JH, et al. Identification of a cholangiocarcinomalike gene expression trait in hepatocellular carcinoma. Cancer Res 2010;70:3034-3041.

18. Lee CH, Hsieh SY, Chang CJ, Lin YJ. Comparison of clinical characteristics of combined hepatocellular-cholangiocarcinoma and other primary liver cancers. J Gastroenterol Hepatol 2013;28:122-127.

19. Zhou YM, Yang JM, Wang B, et al. Combined hepatocellular carcinoma and cholangiocarcinoma: a case report and review of the literature. Hepatobiliary Pancreat Dis Int 2007;6:656-659.

20. Shetty AS, Fowler KJ, Brunt EM, et al. Combined hepatocellularcholangiocarcinoma: what the radiologist needs to know abou biphenotypic liver carcinoma. Abdom Imaging 2014;39:310-322.

21. Brunt EM, Paradis V, Sempoux C, Theise ND. Biphenotypic (hepatobiliary) primary liver carcinomas: the work in progress. Hepat Oncol 2015:2;255273.

22. Fowler KJ, Brown JJ, Narra VR. Magnetic resonance imaging of focal liver lesions: approach to imaging diagnosis. Hepatology 2011;54:2227-2237.

23. Panjala C, Senecal DL, Bridges MD, et al. The diagnostic conundrum and liver transplantation outcome for combined hepatocellularcholangiocarcinoma. Am J Transplant 2010;10:1263-1267.

24. Wells ML, Venkatesh SK, Chandan VS, et al. Biphenotypic hepatic tumors: imaging findings and review of literature. Abdom Imaging 2015;40:2293-2305.

25. Wang J, Wang F, Kessinger A. Outcome of combined hepatocellular and cholangiocarcinoma of the liver. J Oncol 2010;2010.

26. Amin $M$, Chen $L$, Vachhajarani $N$, et al. Independent predictors of overall survival and recurrence in biphenotypic hepatocellular cancer. J Clin Oncol 2014;32(Suppl 3):Abstract 291.

27. Sapisochin G, Fidelman N, Roberts JP, Yao FY. Mixed hepatocellular cholangiocarcinoma and intrahepatic cholangiocarcinoma in patients undergoing transplantation for hepatocellular carcinoma. Liver Transpl 2011;17:934-942.

28. Valle J, Wasan $\mathrm{H}$, Palmer $\mathrm{DH}$, et al. Cisplatin plus gemcitabine versus gemcitabine for biliary tract cancer. N Engl J Med 2010;362:1273-1281.

29. Chi M, Mikhitarian K, Shi C, Goff LW. Management of combined hepatocellular-cholangiocarcinoma: a case report and literature review. Gastrointest Cancer Res 2012;5:199-202.

30. Llovet JM, Ricci S, Mazzaferro V, et al. Sorafenib in advanced hepatocellular carcinoma. N Engl J Med 2008;359:378-390. 
Targeted Therapy in Biphenotypic Tumors

31. Lee CS, Pirdas A. Epidermal growth factor receptor immunoreactivity in gallbladder and extrahepatic biliary tract tumours. Pathol Res Pract 1995;191:1087-1091.

32. Yoon JH, Gwak GY, Lee HS, et al. Enhanced epidermal growth factor receptor activation in human cholangiocarcinoma cells. J Hepatol 2004;41:808-814.

33. Philip PA, Mahoney MR, Allmer C, et al. Phase II study of erlotinib in patients with advanced biliary cancer. J Clin Oncol 2006;24:3069-3074.

34. Lee J, Park SH, Chang HM, et al. Gemcitabine and oxaliplatin with or without erlotinib in advanced biliary-tract cancer: a multicentre, openlabel, randomised, phase 3 study. Lancet Oncol 2012;13:181-188.

35. Malka D, Cervera P, Foulon S, et al. Gemcitabine and oxaliplatin with or without cetuximab in advanced biliary-tract cancer (BINGO): a randomised, open-label, non-comparative phase 2 trial. Lancet Oncol 2014;15:819-828.

36. Hsieh YY, Tzeng $\mathrm{CH}$, Chen $\mathrm{MH}$, et al. Epidermal growth factor receptor R521K polymorphism shows favorable outcomes in KRAS wild-type colorectal cancer patients treated with cetuximab-based chemotherapy. Cancer Sci 2012;103:791-796.

37. Klinghammer K, Knodler M, Schmittel A, et al. Association of epidermal growth factor receptor polymorphism, skin toxicity, and outcome in patients with squamous cell carcinoma of the head and neck receiving cetuximab-docetaxel treatment. Clin Cancer Res 2010;16:304-310.
38. Goncalves A, Esteyries S, Taylor-Smedra B, et al. A polymorphism of EGFR extracellular domain is associated with progression free-survival in metastatic colorectal cancer patients receiving cetuximab-based treatment. BMC Cancer 2008;8:169.

39. Park BK, Paik YH, Park JY, et al. The clinicopathologic significance of the expression of vascular endothelial growth factor-C in intrahepatic cholangiocarcinoma. Am J Clin Oncol 2006;29:138-142.

40. Lubner SJ, Mahoney MR, Kolesar JL, et al. Report of a multicenter phase II trial testing a combination of biweekly bevacizumab and daily erlotinib in patients with unresectable biliary cancer: a phase II Consortium study. J Clin Oncol 2010;28:3491-3497.

41. Zhu AX, Meyerhardt JA, Blaszkowsky LS, et al. Efficacy and safety of gemcitabine, oxaliplatin, and bevacizumab in advanced biliary-tract cancers and correlation of changes in 18-fluorodeoxyglucose PET with clinical outcome: a phase 2 study. Lancet Oncol 2010;11:48-54.

42. Ross JS, Wang K, Catenacci DV, et al. Comprehensive genomic profiling of biliary tract cancers to reveal tumor-specific differences and genomic alterations [abstract]. J Clin Oncol 2015;33(Suppl 3):Abstract 231.

43. Keller JW, Doyle MM, Wang-Gillam A, et al. Analysis of the genomic profile of biphenotypic tumors compared to cholangiocarcinoma and hepatocellular carcinoma [abstract]. J Clin Oncol 2014;32(Suppl):Abstract 226.

44. Enjoji F, Aishima S. Digestive organs: liver: combined hepatocellular and cholangiocarcinoma. Atlas Genet Cytogenet Oncol Haematol 2008;12:409-411. 